\title{
A neurological phenotype in nail patella syndrome (NPS) patients illuminated by studies of murine Lmx1b expression
}

\author{
Jennifer A Dunston ${ }^{1}$, Tyler Reimschisel ${ }^{1}$, Yu-Qiang Ding ${ }^{2,3,4,7}$, Elizabeth Sweeney ${ }^{5}$, \\ Randy L Johnson ${ }^{6}$, Zhou-Feng Chen ${ }^{2,3,4}$ and Iain McIntosh ${ }^{*,}{ }^{\prime}$
}

\begin{abstract}
${ }^{1}$ McKusick-Nathans Institute of Genetic Medicine, Johns Hopkins University, Baltimore MD, USA; ${ }^{2}$ Department of Anesthesiology, Washington University School of Medicine Pain Center, St Louis MO, USA; ${ }^{3}$ Department of Psychiatry, Washington University School of Medicine Pain Center, St Louis MO, USA; ${ }^{4}$ Department of Molecular Biology and Pharmacology, Washington University School of Medicine Pain Center, St Louis MO, USA; ${ }^{5}$ Royal Liverpool Children's Hospital, Alder Hey, Liverpool, UK; ${ }^{6}$ Department of Biochemistry and Molecular Biology, University of Texas MD Anderson Cancer Center, Houston TX, USA
\end{abstract}

Nail patella syndrome (NPS) is an autosomal dominant disorder affecting development of the limb, kidney and eye. NPS is the result of heterozygous loss-of-function mutations in the LIM-homeodomain transcription factor, LMX1B. Recent studies suggest that the NPS phenotype may be more extensive than recognized previously including neurologic and neurobehavioral aspects. To determine whether these findings correlated with the expression of $L m \times 1 b$ during development, an internal ribosomal entry siteLacZ reporter was inserted into the $3^{\prime} U T R$ of the endogenous murine gene. The pattern of $\operatorname{Lm} \times 1 b$ expression during the development of the limb, eye and kidney correlates with the NPS phenotype. Additional sites of expression were observed in the central nervous system (CNS). The effects of the absence of $\operatorname{Lm} \times 1 \mathrm{~b}$ in the CNS were determined in $\operatorname{Im} \times 1 b^{-/-}$mice by histology and immunocytochemistry. $\mathrm{Lm} \times 1 \mathrm{~b}$ is required for the differentiation and migration of neurons within the dorsal spinal cord. The inability of afferent sensory neurons to migrate into the dorsal horn is entirely consistent with diminished pain responses in NPS patients.

European Journal of Human Genetics (2005) 13, 330-335. doi:10.1038/sj.ejhg.5201332

Published online 24 November 2004

Keywords: nail patella syndrome; LMX1B; neuronal migration

Introduction

Nail patella syndrome (NPS, OMIM 161200), or onychoosteodysplasia, is a pleiotropic birth defect exhibiting an autosomal dominant pattern of inheritance. Congenital manifestations include dysplastic nails, absent or hypoplastic patellae, bony projections ('horns') of the ilia and

*Correspondence: Dr I McIntosh, McKusick-Nathans Institute of Genetic Medicine, Johns Hopkins University, 733 N. Broadway/BRB 407, Baltimore, MD 21205, USA. Tel: +1 410955 7948; Fax: + 1410502 5677; E-mail: mcintosh@jhmi.edu

${ }^{7}$ Current address: Laboratory of Neural Development, Institute of Neuroscience, Chinese Academy of Sciences, Shanghai, China.

Received 21 May 2004; revised 2 October 2004; accepted 6 October 2004 dysplasia of the elbows (reviewed in Carbonarna and Alpert, ${ }^{1}$ Bongers et $a l^{2}$ and Sweeney et $a l^{3}$ ). The obvious orthopedic and ectodermal issues have resulted in NPS being regarded as primarily a disorder of connective tissue. However, it has become apparent that proteinuria, resulting from structural abnormalities of the glomerular basement membrane and open-angle glaucoma are constituent parts of the syndrome and may occur at any age. ${ }^{3-5}$ The observation of a phenotype similar to NPS in $\operatorname{lm} \times 1 b^{-/-}$ mice ${ }^{6}$ prompted Dreyer et $a l^{7}$ to consider $L M X 1 B$ as a candidate gene for NPS. Subsequent work has identified approximately 120 distinct loss-of-function mutations. ${ }^{8}$ LMX1B is a member of the LIM-homeodomain family of 
transcription factors that play a variety of roles during development to determine body pattern in vertebrates and invertebrates. ${ }^{9,10}$ Both the expression of Lmx1b during development and the spectrum of abnormalities observed in the $\operatorname{lm} \times 1 b^{-/-}$mouse correlate with the NPS phenotype in terms of the ocular, kidney and limb abnormalities. ${ }^{3,7}$

Recently, in a large clinical study of 123 NPS patients, peripheral neurological symptoms were recognized, described as numbness, tingling or burning sensations of the distal limb, in 25\% (28/110) of patients. ${ }^{3}$ The neurological problems may be related to abnormalities of the dorsal-ventral patterning of the developing limb including abnormal neuronal migration. Cases in which orthopedic abnormalities may have resulted in nerve entrapment were excluded. ${ }^{3}$ Alternatively, the neurological symptoms may result from a primary dysfunction of LMX1B within the sensory circuit. Lmx1b expression has been employed as a marker of neuronal differentiation in the developing spinal cord. ${ }^{11,12}$

To further investigate the role of LMX1B in the neurological symptoms in NPS, the spatiotemporal pattern of Lmx1b was studied using a mouse model with an internal ribosomal entry site (IRES)-LacZ cassette inserted into the $3^{\prime}$ UTR of the gene. The IRES allows for the translation of both the $\mathrm{Lmx} 1 \mathrm{~b}$ and $\beta$-galactosidase proteins from a single transcript. A nuclear localization signal is included to direct $\beta$-galactosidase to the nucleus, thus replicating Lmx1b expression. Using $\beta$-galactosidase staining as a marker of Lmx1b, a dynamic pattern of expression was observed within the developing spinal cord, with expression persisting through adulthood. Analysis of the spinal cord of $\operatorname{lm} \times 1 b^{-/-}$mice revealed deficit in migration of postmitotic neurons to the superficial layers of the dorsal spinal cord, and inability of afferent fibers to penetrate these layers. Detailed neurological examination revealed neurological deficits in 11 of 16 individuals with NPS. A pathophysiological mechanism linking Lmx1b expression to a neurological phenotype in NPS is proposed.

\section{Methods \\ Construction of the $\operatorname{lm} \times 1 b^{3^{\prime} \mathrm{LacZ}}$ mouse}

An IRES-LacZ-floxed PGK-neo cassette ${ }^{13}$ with a nuclear localization signal was inserted into a unique HindIII site in the $3^{\prime} \mathrm{UTR}$ of $\operatorname{lm} \times 1 b$ via homologous recombination in $\mathrm{AB} 1$ ES cells. Two independent lines were injected into $\mathrm{C} 57 \mathrm{Bl} / 6$ blastocysts and the resulting chimeras assayed for germline transmission by mating to $\mathrm{C} 57 \mathrm{Bl} / 6$ females. The presence of the LacZ cassette was determined by PCR followed by Southern analysis using a probe external to the targeting vector. The neo cassette was removed by mating with CMV-cre mice and homozygous lines were established by intercrossing the resultant progeny and selecting for cre-negative homozygotes. The presence of the LacZ cassette does not affect normal function of $\operatorname{Lmx} 1 \mathrm{~b}$, since the LacZ homozygotes are viable and fertile, as were mice that were compound heterozygotes containing the lacZ allele over the null allele (data not shown). The generation of the $\operatorname{lm} \times 1 b^{-/-}$mice was described previously. ${ }^{6}$

\section{$\beta$-galactosidase staining, histology and immunocytochemistry}

$\mathrm{CD}_{1}$ females, used because of their large litter sizes, were crossed with either an $\operatorname{lm} x 1 b^{3^{\prime} \mathrm{LaCZ}}$ heterozygote or homozygote. The day the plug was seen was designated E0.5. Embryos were collected from E9.5 to E15.5 at one-day intervals. Embryos at E14.5 and E15.5 were partially dissected prior to staining. Adult spinal cord was dissected from postnatal day 21 (P21) mice. Tissues were fixed and stained as described. ${ }^{14}$ Transverse sections of the cervical region of the spinal cord were made with a razor blade and digital photographs were taken under a Nikon dissecting microscope. Immunocytochemistry was performed on six $\operatorname{lm} \times 1 b^{-/-}$embryos at each developmental stage, as described previously. ${ }^{15,16}$

\section{Patients and neurological examination}

A focused neurology history and neurological examination was performed on 16 sequential, volunteer individuals with NPS after obtaining appropriate informed consent. The focused neurology questions included those signs and symptoms that have been reported in individuals with NPS, ${ }^{3}$ including sensory deficits and neuropathic pain (ie spontaneous or stimulus-induced burning or electric-like pain that is not in a dermatomal or nerve-specific distribution). A comprehensive bedside neurological examination was also performed, including assessment of cranial nerve function, motor function, sensation, tendon reflexes, cerebellar function and gait. Sensation was evaluated by testing pinprick, cold, vibratory and proprioception. Pinprick and cold sensation assess the function of unmyelinated $\mathrm{A} \delta$ and $\mathrm{C}$ fibers. Pinprick sensation was tested by lightly touching the tips of the subject's fingers or toes with a safety pin. The subject was asked to state when the prick felt sharp as the pinpricks moved proximal up the digits and onto the hands or feet. Cold sensation was tested by asking the subject if a cold tuning fork felt the same on the tips of the digits, the palm or dorsum of the foot, and the proximal extremity. Cranial nerve function was assessed by determination of sensation to pinprick and cold in the face.

\section{Results}

Whole mount staining of $\operatorname{lm} x 1 b^{3^{\prime} L a c Z}$ embryos (Figure $1 \mathrm{a}-\mathrm{e}$ ) indicates that the insertion of the IRES-LacZ cassette does not alter the endogenous pattern of Lmx1b expression. At E9.5, strong expression of Lmx1b is observed in the forelimb bud and midbrain-hindbrain junction (Figure 1a). By E10.5, Lmx1b is also expressed in the hindlimb bud, and expression within the periocular mesenchyme is visible (Figure 1c). Lmx1b continues to be expressed in the limb 
a

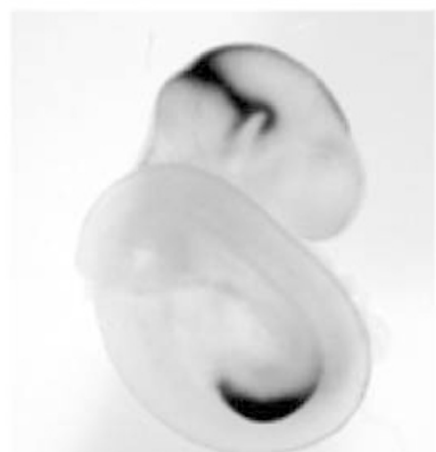

E9.5

d

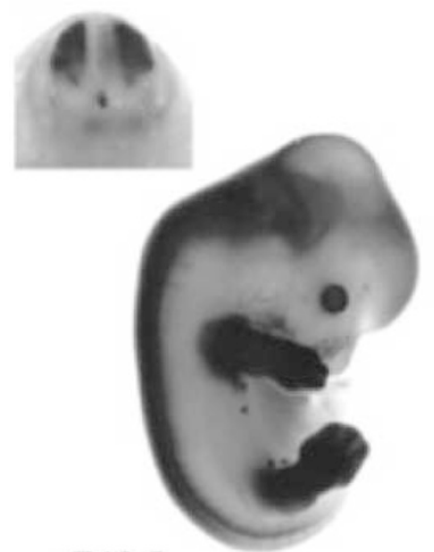

b

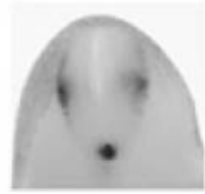

E10.5

e

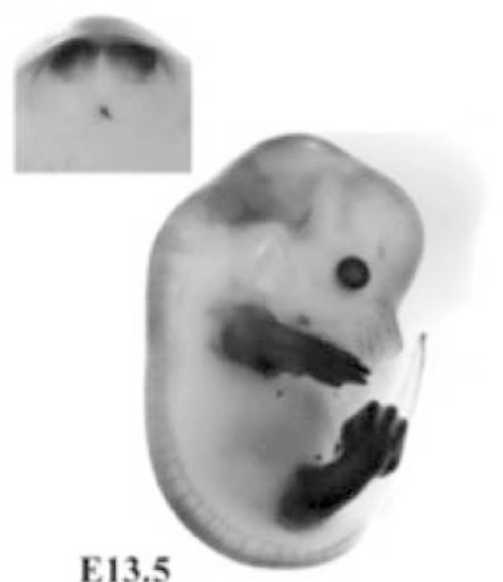

C

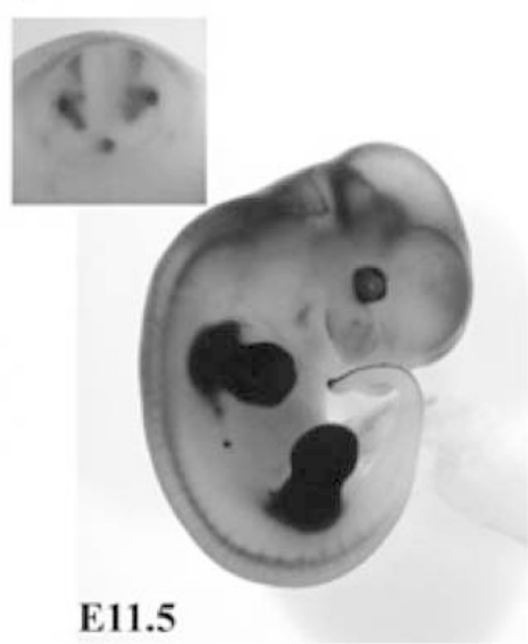

f

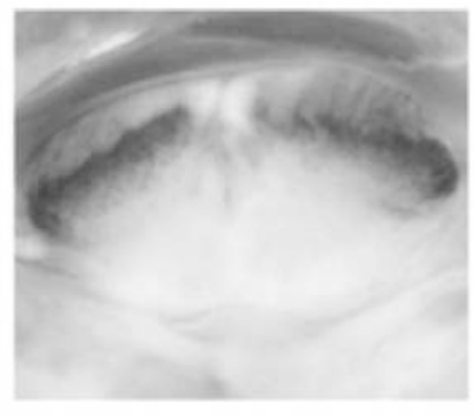

P21

Figure 1 (a-e) Whole-mount $\beta$-galactosidase staining of $I m \times 1 b^{3^{\prime} \text { LacZ }}$ embryos at the stages indicated. Transverse sections of the spinal cord are shown in insets. (f) Transverse section of adult spinal cord (P21).

buds and eye up to E15.5, the latest time point examined. Additionally, expression was detected in the kidney at E14.5 (data not shown). As reported previously, ${ }^{17} \mathrm{Lmx} 1 \mathrm{~b}$ expression in the cranial mesenchyme is visible by E11.5 (Figure 1c). Other regions of expression include mammary gland primordia, from E11.5 to E15.5, as well as vibrissae and sensory follicle primordia, from E11.5 to E15.5 (Figure 1c and d and data not shown). Hair follicle expression is visible from E14.5 (data not shown). On whole mount, spinal cord expression first becomes visible at E10.5 and by E11.5 extends the entire length of the neural tube.

Transverse sections of the whole mount specimens reveal a dynamic pattern of Lmx1b expression within the spinal cord (Figure 1b-e, insets). From E10.5 to E15.5, Lmx1b was expressed within the floorplate. Lateral neural tube expression initiated at E10.5 (Figure 1b, inset). The fate of these neurons remains unknown. A second region of
Lmx1b expression within the neural tube becomes visible at E11.5 (Figure 1c, inset). This population expands and migrates to the dorsal spinal cord through E12.5 and E13.5 (Figure 1d and e insets) and forms the dorsal sensory interneurons. ${ }^{11}$ Expression of Lmx1b within this population is maintained to adulthood (Figure 1f).

Nissl staining has shown that the laminar boundary is indistinct in $\operatorname{lm} x 1 b^{-1-}$ embryos compared with wild type and development of laminae I and II neurons is impaired. ${ }^{18}$ The impaired development of laminae I and II neurons in $\operatorname{lm} x 1 b^{-/-}$embryos appears to delay the innervation of primary cutaneous afferents, as determined by TrkA staining (Figure 2). ${ }^{18}$ At E14.5, Trk + fibers have migrated into the dorsal laminae in wild-type embryos but not in $\operatorname{lm} \times 1 b^{-/}$ - embryos (Figure 2a and b). By E17.5, TrkA + fibers have migrated into the dorsal laminae in $\operatorname{lm} \times 1 b^{-/-}$embryos, but have migrated in a disorganized manner (Figure 2c and d). 


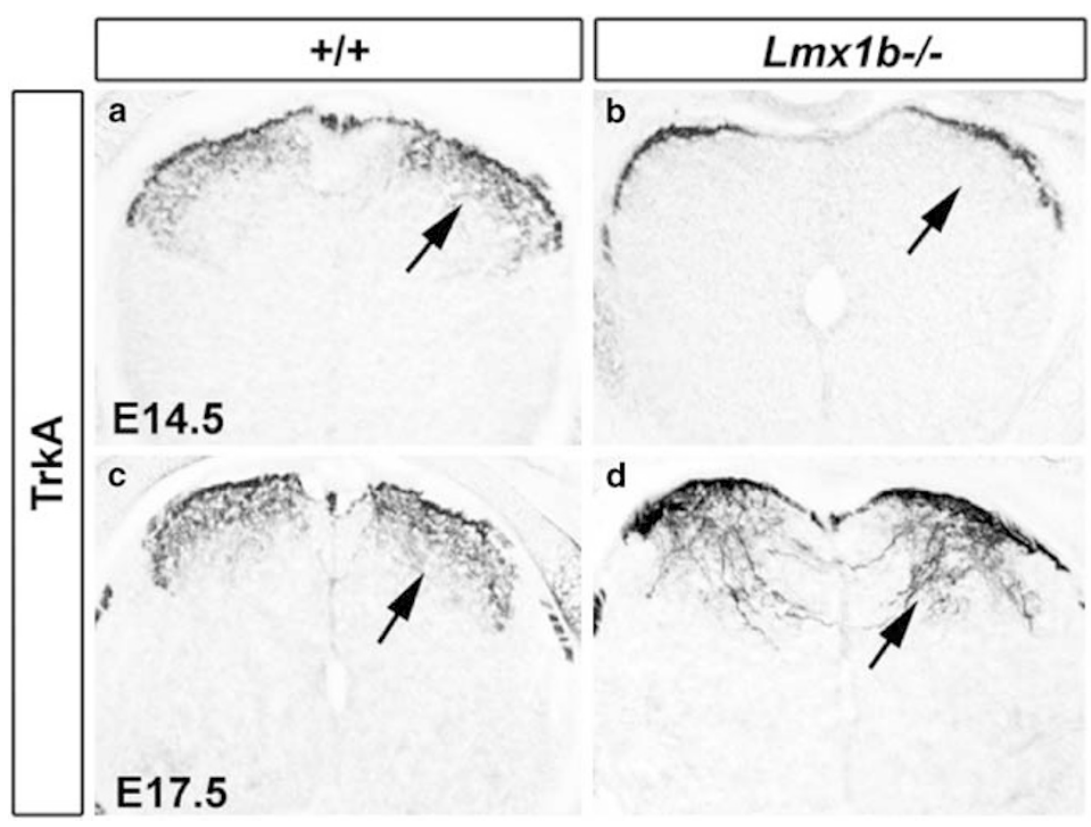

Figure 2 Projection of TrkA + fibers in wild-type (a, c) and $\operatorname{Im} x 1 b^{-/-}$embryos (b, d) at E14.5 (a, b) and E17.5 (c, d). The arrow marks the position of the boundary between laminae I-II and III-V neurons visualized by Nissl staining. ${ }^{\text {is }}$

To determine whether the expression of Lmx1b in the dorsal spinal cord is relevant in consideration of the NPS phenotype, detailed neurological examinations were performed in 16 subjects. The results of the neurological history and examination are shown in Table 1. Numbness or tingling in the face, arms, hands, feet or lower back was reported in 10/16, and 2/16 complained of burning pain. The pain was in the forearm in one individual and in the hands and shins of the other.

In most of the individuals examined, finger pinprick sensation was diminished. (In the absence of calluses or trauma, any reduction in response to pinprick is abnormal.) In a minority of individuals, this deficit was associated with impaired toe and foot pinprick sensation and cold sensation in the hands and feet (see Table 1). When pinprick sensation was impaired, the ventral and dorsal surfaces were equally impaired, and all the digits were usually affected equally. Also, the impairment was typically symmetric between the right and left hands or feet. In two cases, a deficit in sensation was observed in the absence of numbness or tingling. There was no apparent association between neurological symptoms and LMX1B mutations: symptoms were noted in individuals with missense, nonsense or frameshift mutations, and symptoms were as variable between individuals with the same mutation as among the cohort as a whole.

Cranial nerve function and cerebellar function were within normal limits. Weakness and hyporeflexia was noted in three individuals, but was likely secondary to joint disease and orthopedic procedures. Gait was abnormal in only one individual who walked with a cane due to severe orthopedic problems. Proprioception in the thumb and great toe was diminished in one individual. Vibration sensation in the great toe was diminished in five individuals. This may be associated with NPS, but the fibers that transmit vibration sensation do not synapse onto laminae I and II (see below). However, this deficit is not rare in the general population, particularly in older individuals.

\section{Discussion}

The pattern of $\operatorname{lm} x 1 b$ expression observed in limb, eye and kidney correlates with the recognized signs and symptoms of NPS. The relevance to the NPS phenotype of Lmx1b expression in cranial mesenchyme, mammary gland primordia, hair follicles and vibrissae remains to be determined, although poor breast development has been reported in some women with NPS, and a characteristic hairline described. ${ }^{3}$ Preliminary evidence suggests that ADHD may be a constituent part of NPS, associated with the requirement for Lmx1b in the development of mesencephalic dopaminergic neurons (McIntosh et al, in preparation). ${ }^{19,20}$

TrkA + fibers are nociceptive sensory neurons with cell bodies located in the dorsal root ganglia and have axons that project into the dorsal laminae of the spinal cord (reviewed by Snider and McMahon ${ }^{21}$ ). Although the migration of the TrkA afferents into the spinal cord of $\operatorname{lm} \times 1 b^{-/-}$mice is abnormal, this deficit most likely reflects a noncell autonomous role of Lmx1b in the migration of the TrkA afferents since Lmx1b is not expressed in the dorsal root ganglion. Alternatively, the expression of Lmx1b in the outer laminae of the developing spinal cord may be 
Table 1 Neurological findings in NPS patients

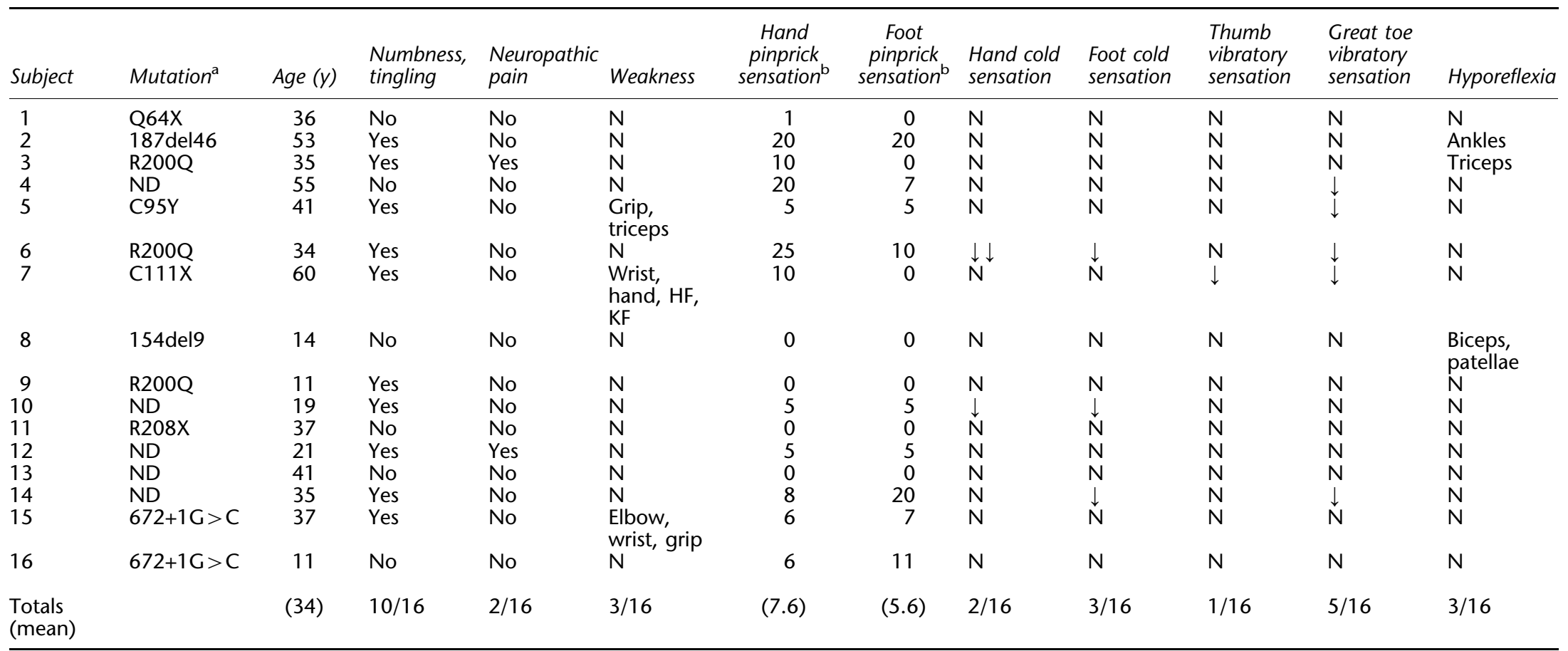

$\mathrm{N}=$ normal; $\downarrow=$ diminished; $\mathrm{HF}=$ hip flexion; $\mathrm{KF}=$ knee flexion; $\mathrm{ND}=$ not determined

${ }^{a}$ Mutations were identified as described. ${ }^{8}$

(a)

reported as cumulative score of impairment for all digits on one hand/foot, where $0=$ no deficit, $1=$ impairment distal to DIP joint, $2=$ impairment between DIP and PIP joints; 3 =impairment between DIP and MCP joints; $4=$ impairment between MCP joint and wrist/ankle; $5=$ impairment to wrist/ankle. DIP=distal-interphalangeal joint, $\mathrm{PIP}=$ proximal - interphalangeal joint, $\mathrm{MCP}=$ metacarpal - phalangeal joint. 
necessary to establish the proper developmental cues for the projection of TrkA fibers into the dorsal horn.

Pain and temperature modalities are carried by lightly myelinated $\mathrm{A} \delta$ and unmyelinated $\mathrm{C}$ fibers. ${ }^{22,23}$ The $\mathrm{A} \delta$ fibers are responsible for sharp pain (ie pinprick response) and are found in higher numbers in the distal limb relative to the proximal limb. ${ }^{23}$ Dysfunction of the $\mathrm{A} \delta$ and $\mathrm{C}$ fibers causes impaired pinprick and cold sensation, and the patient will frequently complain of numbness, tingling and neuropathic pain. With regard to the NPS phenotype, there is a high incidence of tingling or numbness in individuals with NPS (Table 1$){ }^{3}$ Two individuals complained of neuropathic pain. Pinprick sensation was diminished in a significant number of individuals examined. The deficit was noted in the hands more than the feet, but not in the arms, legs or face. A minority of these individuals also had deficits in cold sensation in the hands and feet but not in the face. Notably, there was no difference between sensitivity across either the dorsoventral or anterior-posterior axes, which would correlate with the expression pattern of Lmx1b. The degree of impairment may be correlated with age since the mildest symptoms were seen in adolescents, and two of the highest scores in subjects over 50 years, but a larger (preferably longitudinal) study is required to address this issue.

Vibration sensation and proprioception are mediated by myelinated fibers. Since these modalities were preserved in most of the affected individuals, it is likely that the observed neurological deficit results from abnormality of the distal, unmyelinated fibers of the hands and feet. Furthermore, the impaired sensation to pinprick and cold temperature did not follow a specific nerve or dermatome pattern. Therefore, it is unlikely that these findings are due to nerve compression secondary to bone degeneration.

The $\mathrm{A} \delta$ and $\mathrm{C}$ fibers synapse in laminae I and II within the spinal cord. In $L m \times 1 b$ mutant mice, differentiation of laminae I and II neurons and cutaneous afferent ingrowth from the dorsal root are impaired (Figure 2). ${ }^{18}$ The cell bodies of $\mathrm{A} \delta$ and $\mathrm{C}$ neurons are located in the dorsal root ganglia, where Lmx1b is not expressed, supporting the hypothesis that the phenotype is the result of a requirement of Lmx1b-specified interneurons for TrkA+ fiber innervation and not a defect in the development of the neurons per se. Therefore, in individuals with NPS, deficient $\mathrm{A} \delta$ and $\mathrm{C}$ fiber projection into laminae I and II may be associated with the neuropathic pain, tingling, and impaired pinprick and cold sensations observed in this study.

\section{Acknowledgements}

We would like to thank Drs Andrew McCallion and Julie Hoover-Fong for helpful discussion and critical review of the manuscript, and Dr Lori E. Kotch for helpful discussions and access to microscopes. This work was supported in part by NIH Grants AR44702 (IM), EY12311 (RLJ) and NS43968 (ZFC), and grants from the Birth Defects Foundation and the Skeletal Dysplasia Group (ES).

\section{References}

1 Carbonara P, Alpert M: Hereditary osteo-onycho-dysplasia (HOOD). Am J Med Sci 1964; 248: 139-151.

2 Bongers EM, Gubler MC, Knoers NV: Nail-patella syndrome. Overview on clinical and molecular findings. Pediatr Nephrol. 2002; 17: 703-712.

3 Sweeney E, Fryer A, Mountford R, Green A, McIntosh I: Nail patella syndrome: a review of the phenotype aided by developmental biology. J Med Genet 2003; 40: 153-162.

4 Bodziak KA, Hammond WS, Molitoris BA: Inherited diseases of the glomerluar basement membrane. Am J Kid Dis 1994; 23 : 605-618.

5 Lichter PR, Richards JE, Downs CA, Stringham HM, Boehnke M, Farley FA: Cosegregation of open-angle glaucoma and the nailpatella syndrome. Am J Ophthalmol 1997; 124: 506-515.

6 Chen H, Lun Y, Ovchimnikov D et al: Limb and kidney defects in $L m \times 1 b$ mice suggest an involvement of $L M X 1 B$ in human nailpatella syndrome. Nat Genet 1998; 19: 51-55.

7 Dreyer SD, Zhou G, Baldini A et al: Mutations in $L M X 1 B$ cause abnormal skeletal patterning and renal dysplasia in nail patella syndrome. Nat. Genet 1998; 19: 47-50.

8 Dunston JA, Hamlington JD, Zaveri J et al: The human LMX1B gene: transcription unit, promoter and pathogenic mutations. Genomics 2004; 84: 565-576.

9 Dawid IB, Toyama R, Taira M: LIM domain proteins. C R Acad Sci III 1995; 318: 295-306.

10 Curtiss J, Heilig JS: DeLIMiting development. Bioessays 1998; 20: $58-69$.

11 Gross MK, Dottori M, Goulding M: Lbx1 specifies somatosensory association interneurons in the dorsal spinal cord. Neuron 2002; 34: 535-549.

12 Muller $\mathrm{T}$, Brohmann $\mathrm{H}$, Pierani A et al: The homeodomain factor lbx1 distinguishes two major programs of neuronal differentiation in the dorsal spinal cord. Neuron 2002; 34: $551-562$.

13 Bi W, Deng JM, Zhang Z, Behringer RR, de Crombrugghe B: Sox9 is required for cartilage formation. Nat Genet 1999; 22: $85-89$.

14 Nonchev SG, Maconochie MK: Spatial analysis of gene expression; in Jackson IJ, Abbott CM (eds): Mouse Genetics and Transgenics: A Practical Approach. Oxford: Oxford University Press, 2000, pp 61-86.

15 Chen ZF, Paquette AJ, Anderson DJ: NRSF/REST is required in vivo for expression of multiple neuronal target genes during embryogenesis. Nat Genet 1998; 20: 136-142.

16 Chen ZF, Rebelo S, White F et al: The paired homeodomain protein DRG11 is required for the projection of cutaneous sensory afferent fibers to the dorsal spinal cord. Neuron 2001; 31: $59-73$.

17 Chen H, Ovchinnikov D, Pressman CL, Aulehla A, Lun Y, Johnson RL: Multiple calvarial defects in $\operatorname{lm} x 1 b$ mutant mice. Dev Genet 1998; 22: 314-320.

18 Ding YQ, Kania A, Johnson RL, Chen ZF: Lmx1b controls the differentiation and migration of the superficial dorsal horn neurons of the spinal cord. Development 2004; 131: $3693-3703$

19 Smidt MP, Asbreuk CH, Cox JJ, Chen H, Johnson RL, Burbach JP: A second independent pathway for development of mesencephalic dopaminergic neurons requires Lmx1b. Nat Neurosci 2000; 3: $337-341$

20 McIntosh I, Tierny E, Bukelis I, Marsh L: Behavioral phenotype of nail-patella syndrome. Am J Hum Genet 2001; 69S: A571.

21 Snider WD, McMahon SB: Tackling pain at the source: new ideas about nociceptors. Neuron 1998; 20: 629-632.

22 Goetz CG, Pappert EJ (eds): Textbook of Clinical Neurology. Philadelphia, PA: WB Saunders, 1999, 1st edn, pp 315-349.

23 Agostino R, Cruccu G, Iannetti G, Romaniello A, Truini A, Manfredi M: Topographical distribution of pinprick and warmth thresholds to $\mathrm{CO}_{2}$ laser stimulation on the human skin. Neurosci Lett 2000; 285: 115-118. 